\title{
Comparison of prediction power of three multivariate calibrations for estimation of leaf anthocyanin content with visible spectroscopy in Prunus cerasifera
}

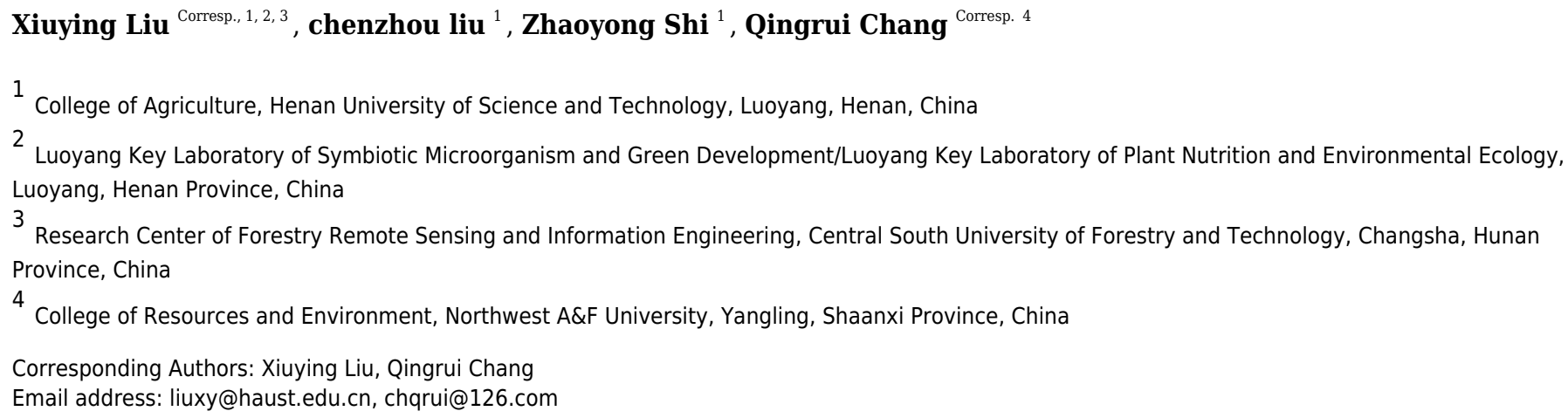

The anthocyanin content in leaves can reveal valuable information about a plant's physiological status and its responses to stress. Therefore, it is of great value to accurately and efficiently determine anthocyanin content in leaves. The selection of calibration method is a major factor which can influence the accuracy of measurement with visible and near infrared (NIR) spectroscopy. Three multivariate calibrations including principal component regression (PCR), partial least squares regression (PLSR), and backpropagation neural network (BPNN) were adopted for the development of determination models of leaf anthocyanin content using reflectance spectra data (450-600 nm) in Prunus cerasifera and then the performance of these models was compared for three multivariate calibrations. Certain principal components (PCs) and latent variables (LVs) were used as input for the back-propagation neural network (BPNN) model. The results showed that the best PCR and PLSR models were obtained by standard normal variate (SNV), and BPNN models outperformed both the PCR and PLSR models. The coefficient of determination $\left(R^{2}\right)$, the root mean square error of prediction $\left(\mathrm{RMSE}_{\mathrm{p}}\right)$, and the residual prediction deviation (RPD) values for the validation set were $0.920,0.274$, and 3.439 , respectively, for the BPNN-PCs model, and 0.922, 0.270, and 3.489, respectively, for the BPNN-LVs model. Visible spectroscopy combined with BPNN was successfully applied to determine leaf anthocyanin content in $P$. cerasifera and the performance of the BPNN-LVs model was the best. The use of the BPNN-LVs model and visible spectroscopy showed significant potential for the nondestructive determination of leaf anthocyanin content in plants. 
1 Comparison of prediction power of three multivariate calibrations for estimation of leaf

2

3

4

5

$6{ }^{1}$ College of Agronomy, Henan University of Science and Technology, Luoyang, 471023, Henan

$8 \quad{ }^{2}$ Luoyang Key Laboratory of Symbiotic Microorganism and Green Development/Luoyang Key

9 Laboratory of Plant Nutrition and Environmental Ecology, Luoyang, 471023, Henan Province,

10 China;

$11{ }^{3}$ Research Center of Forestry Remote Sensing and Information Engineering, Central South

University of Forestry and Technology, Changsha, 410004, Hunan Province, China;

${ }^{4}$ College of Resources and Environment, Northwest A\&F University, Yangling, 712100, Shaanxi

Province, China

$16 *$ Corresponding author:

17 Xiuying Liu

E-mail: csfulxy@126.com

Qingrui Chang

$$
\text { E-mail: chqrui@126.com }
$$




\section{Abstract}

23

24

The anthocyanin content in leaves can reveal valuable information about a plant's physiological status and its responses to stress. Therefore, it is of great value to accurately and efficiently determine anthocyanin content in leaves. The selection of calibration method is a major factor which can influence the accuracy of measurement with visible and near infrared (NIR) spectroscopy. Three multivariate calibrations including principal component regression (PCR), partial least squares regression (PLSR), and back-propagation neural network (BPNN) were adopted for the development of determination models of leaf anthocyanin content using reflectance spectra data $(450-600 \mathrm{~nm})$ in Prunus cerasifera and then the performance of these models was compared for three multivariate calibrations. Certain principal components (PCs) and latent variables (LVs) were used as input for the back-propagation neural network (BPNN) model. The results showed that the best PCR and PLSR models were obtained by standard normal variate (SNV), and BPNN models outperformed both the PCR and PLSR models. The coefficient of determination $\left(\mathrm{R}^{2}\right)$, the root mean square error of prediction $\left(\mathrm{RMSE}_{\mathrm{p}}\right)$, and the residual prediction deviation (RPD) values for the validation set were $0.920,0.274$, and 3.439 , respectively, for the BPNN-PCs model, and 0.922, 0.270, and 3.489, respectively, for the BPNN-LVs model. Visible spectroscopy combined with BPNN was successfully applied to determine leaf anthocyanin content in P. cerasifera and the performance of the BPNN-LVs model was the best. The use of the BPNN-LVs model and visible spectroscopy showed significant potential for the nondestructive determination of leaf anthocyanin content in plants. 
43 Keywords Anthocyanin content, Reflectance spectra; Back-propagation neural network, Partial

44 least squares analysis, Principal component analysis

45

46 


\section{INTRODUCTION}

Anthocyanins are a large group of water soluble flavonoid pigments (Strack, 1997; Iwashina, 2000), the common pigment, that occur in all tissues of higher plants, including the leaves, stems, roots, flowers, and fruits. They are responsible for a wide range of plant colors, such as blue, purple, violet, magenta, red and orange (Fennema, 1998; Lai, 2019), but they often appear red (Gould et al., 1995; Van den Berg \& Perkins, 2005; Gould, Davies \& Winefield, 2009). Anthocyanins serve many functions, including pollinator attraction, as protectants (Gould, Davies \& Winefield, 2009), as antioxidants (Gould, McKelvie \& Markham, 2002, Yang et al., 2017), and as osmoprotectants (Chalker-Scott, 1999). These compounds also play a photo-protective role (Liakopoulos et al., 2006), and act as optical barriers (Close \& Beadle, 2003; Solovchenko \& Merzlyak, 2008). A number of environmental stresses, such as strong light, low temperature, UV-B irradiation, wounding, drought, bacterial and fungal infections, deficiencies in nitrogen, phosphorus and potassium, and certain herbicides and pollutants can result in the significant accumulation of anthocyanins (Saure, 1990; Garriga et al., 2014; Zhang et al., 2018), which are thus often referred to as "stress pigments" (Chalker-Scott, 1999). In addition, anthocyanins accumulate transiently in juvenile and senescing leaves in many plant species under unfavorable conditions (Karageorgou \& Manetas, 2006; Merzlyak et al., 2008; Zeliou, Manetas \& Petropoulou, 2009; Garriga et al., 2014). Thus, anthocyanin content can serve as an indicator of leaf senescence and environmental stresses in many plant species (Neill \& Gould, 1999; Gitelson \& Merzlyak, 2004), so the accurate detection and quantitative assessment of anthocyanin can provide important and valuable information about the physiological responses and adaptation of 
68

69

plants to environmental stresses (Gamon \& Surfus, 1999; Gitelson, Chivkunova \& Merzlyak, 2009;

Ustin et al., 2009). The traditional method to determine anthocyanin content has been the wetchemical method (Gitelson \& Merzlyak, 2004; Gitelson, Merzlyak \& Chivkunova, 2001; Steele et al., 2009). This method is laborious, time-consuming, expensive, and requires the destruction of leaves for measurement (Solovchenko et al., 2001; Merzlyak, Solovchenko \& Gitelson, 2003; Steel et al., 2009). In addition, this measurement method does not allow the measurement of changes in pigments over time in a single leaf (Garriga et al., 2014).

Visible and near infrared reflectance (Vis/NIR) spectroscopy has been widely used in recent decades to measure pigments. The spectral absorbance properties of pigments are present in the reflectance spectra of leaves, thus measurements of reflected radiation can be used as a nondestructive method to quantify pigments (Blackburn, 2007). Non-destructive technology based on spectrum analysis has several advantages over conventional methods, including simplicity, sensitivity, inexpensive, good reliability of the method, and high performance (Viscarra Rossel, McGlynn \& McBratney, 2006; Kira, Linker \& Gitelson, 2015; Nagy, Riczu \& Tamás, 2016). This technique can be applied at different spatial scales and in a large number of samples (Viña \& Gitelson, 2005; Lobos et al., 2014). Compared with traditional multispectral techniques, hyperspectral remote sensing, which provides a continuous reflectance spectrum with narrow wavebands, can characterize vegetation and provide a considerably greater amount of information than what can be obtained using traditional multispectral techniques (Goetz, 2009; Mulla, 2013). Therefore, recent research has focused on developing techniques to analyze plant spectra to more accurately quantify pigment concentrations (Blackburn, 2007). Most research has focused on the 
89

90

91

92

93

94

95

96

97

98

estimation of chlorophyll and carotenoid content, but little is known about anthocyanin estimation from reflectance spectra, and most pigment measurement studies utilize linear/or simple nonlinear models (Chappelle et al., 1992; Gitelson, Merzlyak \& Chivkunova, 2001; Blackburn, 2007). For anthocyanins, various models (called vegetation indices) have been developed based on the spectral information (e.g. Gitelson, Merzlyak \& Chivkunova, 2001; Gitelson \& Merzlyak, 2004; Gitelson et al., 2006; Gitelson, Chivkunova \& Merzlyak, 2009; Van den Berg \& Perkins, 2005; Merzlyak et al., 2008; Steele et al., 2009; Garriga et al., 2014; Liu et al.,2015; Manjunath, Shibendu \& Dhaval, 2016).

The empirical statistical approach is a main approach to building relationships between spectral data and biochemical or biophysical parameters. The modern spectral technique (especially hyperspectral data) generally produces abundant data for the analyzed object. However multi-collinearity is a common problem inherent to hyperspectral dataset (Mirzaie et al., 2014). There are convoluted interrelations between individual values of reflectance and biological properties (Garriga et al., 2014). Moreover univariate regression models based on vegetation indices, which typically use two to three bands, cannot capture the intrinsic relationships between the observed remote sensing data (especially hyperspectral data) and biochemical or biophysical parameters of interest (Camps-Valls, 2006). Furthermore, the selection of calibration method is a main factor influencing measurement accuracy with visible and near infrared reflectance (Vis/NIR) spectroscopy (Mouazen et al., 2010). Hence, it is important to use multivariate calibration algorithms to better develop the relationship between spectral data and the analyzed object and compare predictive performance (Mouazen et al., 2010, Li \& He, 2010). Linear and 
110

111

112

113

114

115

116

117

118

119

120

121

122

123

124

125

126

127

128

129

130

nonlinear multivariate calibration techniques include principal component regression (PCR), partial least squares regression (PLSR), and back-propagation neural network (BPNN), and have been widely and successfully applied in spectra analysis (Vasques, Grunwald \& Sickman, 2008; Liu et al., 2008; Atzberger et al., 2010; Li \& He, 2010; Kinoshita et al., 2011; Mirzaie et al., 2014; Gomes et al., 2017; Wang et al., 2018). The PCR and PLSR analyses are the most common techniques for spectral calibration and prediction (Viscarra Rossel, McGlynn \& McBratney, 2006), and these two methods may reduce the effect of the multi-collinearity problem. The artificial neural network (ANN) has many advantages such as nonlinear mapping, high accuracy for learning, and good robustness (Atkinson et al., 1997; Keiner \& Yan, 1998). For this reason, artificial neural networks are increasingly used in visible and near infrared reflectance (Vis/NIR) spectroscopy (Liu et al., 2008; Gomes et al., 2017).

Prunus cerasifera (P. cerasifera), commonly called cherry plum, is a Prunus deciduous small trees that is natives to western Asia and the Caucasus. Its leaves contain high amounts of anthocyanins, which makes them appear purple. P. cerasifera has become a very popular ornamental landscape tree in large part because its showy purple foliage retains excellent color throughout the growing season. The leaves of $P$. cerasifera exhhibit a wide range of anthocyanin contents, making $P$. cerasifera a good object to study the content of leaf anthocyanins in plants. To the best of our knowledge, no work has explored the combination of PLSR or PCR with ANN for the analysis of leaf anthocyanin content of $P$. cerasifera using visible spectroscopy (450-600 $\mathrm{nm})$.

In this study, the leaf anthocyanin content of $P$. cerasifera was investigated with visible 
131 spectroscopy based on three multivariate calibrations. The objectives of the present work were: (1)

132 to investigate the feasibility of using visible spectroscopy to determine the anthocyanin content in

133 P. cerasifera leaves; (2) to determine the optimal spectral pretreatments after the comparison of

134 Savitzky-Golay (SG) smoothing, standard normal variate (SNV), multiplicative scattering

135 correction (MSC), first derivative(1-Der), standard normal variate in combination with

136 transformed baseline (SNV+TB), Savitzky-Golay smoothing in combination with first derivative

137 (SG+1-Der), and multiplicative scattering correction in combination with first derivative (MSC +1 -

138 Der); (3) to develop the best calibration models to estimate the leaf anthocyanin content in $P$.

139 cerasifera comparing the prediction power of principal component regression (PCR), partial least

140 squares regression (PLSR), and back-propagation neural network (BPNN). The results of this

141 study are a preliminary step forward for improving monitoring of the growing status and biological

142 parameters of plants using spectroscopic techniques. 
143

144

145

146

147

148

149

150

151

152

153

154

155

156

157

158

159

160

161

162

163

\section{Materials and methods}

\section{Leaf samples}

In total, 456 pieces of $P$. cerasifera leaves were collected from the Northwest A \& F University campus between March and May of 2015. These leaves, ranging in color from dark green with little red to completely red, were picked from $P$. cerasifera of different ages and oriented in different directions from the stem. After detachment, the leaves were immediately sealed in plastic bags with a small amount of water, labeled as different samples, and then placed on ice for transport to the laboratory. Healthy and homogeneously colored leaves without visible symptoms of damage were used for experiments.

\section{Laboratory analyses of anthocyanin content}

The anthocyanin content was quantitatively measured from the same leaf samples used for reflectance measurement. Several small pieces were cut from the leaves and then $0.15 \mathrm{~g}$ of samples were extracted with $0.1 \mathrm{~mol} \mathrm{~L}^{-1}$ hydrochloric acid methanol solution using the soaking extraction method. For total anthocyanin extraction, 24 hours of soaking time was performed. The resulting extracts were immediately assayed spectrophotometrically, and the anthocyanin content was expressed as a function of leaf amount (i.e., $\mu \mathrm{mol} \mathrm{g}^{-1}$ ). The methods used are described in detail in the literature (Xiong et al., 2003).

\section{Spectrum measurement and pretreatment}

The reflectance spectra of the leaves were measured with a SVC HR-1024i spectrophotometer (Spectra Vista Corporation, USA) equipped with a SVC reflectance probe and interfaced with a personal computer. During measurement, an internal tungsten halogen lamp provided artificial 
164 illumination. The HR-1024i spectrophotometer measures radiance with a spectral resolution of 3.5

$165 \mathrm{~nm}$ in a wavelength range of 350 to $1000 \mathrm{~nm}$. Before measuring the reflectance spectra of the

166 leaves reference measurements were made by rotating the sample holder plate to position the white

167 reference panel facing the probe window. Target measurements were then taken by inserting a leaf

168 between the sample holder plate and the window. For accurate measurement of the reflectance of

169 the leaves, three reflectance measurements were acquired for each leaf and each sample included

170 four leaves of the same color. Thus, average values were calculated of twelve spectra sample to

171 establish a single representative reflectance spectrum.

172 The anthocyanin absorption peaks in situ were around $540-550 \mathrm{~nm}$ in the visible/near-

173 infrared (Vir/NIR) rang (Gitelson, Merzlyak \& Chivkunova, 2001; Merzlyaket et al., 2008). The

analysis showed a high correlation between total anthocyanin content and reflectance spectra

between 350 and $600 \mathrm{~nm}$, and relative low correlation at the other wavebands (Fig 1). Signals in

the first $100 \mathrm{~nm}$ were removed to avoid a low signal-to-noise ratio. Finally, only wavelength bands

between 450 and $600 \mathrm{~nm}$, which avoided the effect of leaf structure and the strongest absorption

of chlorophyll and water, were employed for the calculations.

To remove system noises and external disturbances and to select the best pretreatment

method, some pretreatments were performed on the spectra and the results were compared (Liu et

software (Spectra Vista Corporation, USA). Overlapping detector data were removed, and then

resampling in $1 \mathrm{~nm}$ intervals was performed. Next, seven types of pretreatments were applied and 
185 Golay smoothing (SG), first derivative (1-Der), standard normal variate combined with 186 transformed baseline (SNV+TB), multiplicative scattering correction combined with first 187 derivative (MSC+1-Der), and Savitzky-Golay smoothing combined with first derivative (SG+1Der). SNV, MSC, and SG smoothing were applied to remove the multiplicative effects of scattering, random noise, and spectral baseline shift (Chu, Yuan \& Lu, 2004; Zhao, Qu \& Cheng 2004; Liu et al., 2008; Bao et al., 2012). The first derivative pretreatment method was applied to decrease the baseline shift (Liu et al., 2008). The raw reflectance spectra and preprocessed spectra of $P$. cerasifera leaves are shown in Figures $2 \mathrm{a}-\mathrm{h}$. All pre-processing steps were implemented using the Unscrambler 9.7 (Camo Inc., Oslo, Norway).

Establishment of calibration models

Principal component regression

Principal component regression (PCR) is a method to relate variations in a response variable

(Y-variable) to the variations of several predictors (X-variables), with explanatory or predictive

purposes. This method performs particularly well when the various X-variables express common information with a high amount of correlation, or even collinearity (Martens \& Naes, 1989). The optimal number of principal components (PCs) for a model was determined by examining a plot

of leave-one-out cross-validation residual variance against the number of loadings (Mouazen et al., 2010).

Partial least squares regression

Partial least squares (PLS) analysis is a bilinear regression method (Arana, Jaren \& Arazuri, 
He, 2008; Wu et al., 2011; Zhang et al., 2018). Partial least-squares regression can reduce data

207

208

209

210

211

212

213

214

215

216

217

218

219

220

221

222

223

224

225

226

noise and computation time, with only minor loss of the information contained in the original

variables. The main procedure is to extract the PLS factors and determine the linear

correlationships between the PLS factors and chemical constituents. In the development of the

PLS model, leave-one-out cross-validation was used to evaluate the quality and to prevent

overfitting of the calibration model (Mouazen et al., 2010). All calculations of the PCR and PLSR

were also implemented based on the Unscrambler V9.7.

\section{Back-propagation neural network}

The most popular neural network is BPNN, a type of nonlinear neural network used to solve classification and regression problems. BPNN models usually provide better results than traditional statistical methods. However, extreme long training time requirements and over-fitting

are two main limitations of ANN calibration when using raw spectral data points or when too many spectral data points are selected as inputs (Mouazen et al., 2010). Many studies have shown that adopting PCs or LVs as input for BPNN is an effective way to reduce computation resources and improve the robustness of ANN calibration (He et al., 2006; Janik et al., 2007; Mouazen et al., 2010; Mirzaie et al., 2014). Hence, in this study BPNN analyses were performed using LVs obtained from PLSR (BPNN-LV) and PCs obtained from PCA (BPNN-PC). The first five PCs (spectra preprocessed by SNV) were considered as input variables in this study, since they could explain nearly $95 \%$ of the variance. The first five LVs (spectra preprocessed by SNV) also were applied as input variables of the BPNN model, as the residual variance was the first minimum value (Brown et al., 2005). 
and one output layer (one node) is usually applied for spectral calibration and prediction (Liu et

Therefore a simple-hidden-layer neural network was used in this study to estimate the anthocyanin

content in $P$. cerasifera leaves. Each node in ANN represents a "neuron", and is associated with a

transfer (activation) function that sums the outputs from that node and passes them to the next

layer in the network. The tan-sigmoid function and a linear function were respectively adopted in

the hidden and in the output layers. The numbers of neurons in the hidden layer was optimized by

trial-and-error. For network training, we used Levenberg-Marquardt (TRAINLM), and the early

stopping technique was used to avoid overfitting problems (Demuth, Beale \& Hagan, 2010;

Mirzaie et al., 2014). All BPNN calculations were implemented using the Neural Networks

toolbox of MATLAB. The theory of ANN has been described previously (He et al., 2006). During

training, the number of nodes in the hidden layer was constantly readjusted. When the number of

nodes of the hidden layer was set at five, a very good result was achieved. In this way, the BPNN

model for anthocyanin content was obtained. The structure contained one input layer with five

modes, and the hidden layer contained five nodes and one output node.

To ensure that the calibration or validation set included samples that covered the complete set (76) and the remaining one-third of the samples were considered the validation set (38). 
248 Therefore, each sample was only used in either the calibration or the validation sets, but not both

249 sets. To compare the performances of different calibration models, the same calibration and

250 validation sets were used to test all of the models. Previous studies have assessed the accuracy and

251 the estimating performance of different models in terms of absolute prediction accuracy (RMSE),

252 the coefficient of determination $\left(\mathrm{R}^{2}\right)$, and the residual prediction deviation (RPD) (Saeys, Mouazen

253 \& Ramon, 2005; Viscarra Rossel, McGlynn \& McBratney, 2006; Vasques, Grunwald \& Sickman,

254 2008; Mouazen et al., 2010; Kinoshita et al., 2011; Hu, 2013; Du et al., 2013; Mirzaie et al., 2014;

255 Gomes et al., 2017). In this study, the performance of all models was evaluated by the following

256 indices: the coefficients of determination of calibration $\left(\mathrm{R}_{\text {cal }}^{2}\right)$ and validation $\left(\mathrm{R}^{2}\right.$ val $)$, the root mean

257 square errors of calibration $\left(\mathrm{RMSE}_{\mathrm{c}}\right)$ and validation $\left(\mathrm{RMSE}_{\mathrm{p}}\right)$, and the residual prediction

258 deviations of calibration $\left(\mathrm{RPD}_{\text {cal }}\right)$ and validation $\left(\mathrm{RPD}_{\mathrm{val}}\right)$. The detailed formulas of these indices

259 are as published previously $(H u, 2013)$. Based on experience and previous reports (Viscarra

260 Rossel, McGlynn \& McBratney, 2006; Saeys, Mouazen \& Ramon, 2005), the R ${ }^{2}$ and RPD values

261 were classified as follows: $\mathrm{R}^{2}<0.5$ with $1.0 \leq \mathrm{RPD}<1.4$ indicates poor models/predictions able

262 to distinguish only high and low values; $0.5 \leq \mathrm{R}^{2}<0.65,1.4 \leq \mathrm{RPD}<1.8$ indicates fair

263 models/predictions which can be used for assessment and correlation; $0.65 \leq \mathrm{R}^{2}<0.80,1.8 \leq \mathrm{RPD}$

$264<2.0$ indicates good models/predictions where quantitative predictions are possible; $0.80 \leq \mathrm{R}^{2}<$

$0.90,2.0 \leq \mathrm{RPD}<2.5$ indicates very good quantitative models/predictions, and $\mathrm{R}^{2} \geq 0.90, \mathrm{RPD} \geq$

RPD values, and lower RMSE values.

Results 
269

270

271

272

273

274

275

276

277

278

279

280

281

282

283

284

285

286

287

288

289

\section{Features of spectra}

The raw reflectance spectra of $P$. cerasifera leaves are shown in Figure $2 \mathrm{a}$. The processed spectra, SG, SNV, MSC, 1-Der, SNV+TB, SG+1-Der, and MSC+1-Der values are shown in Figures $2 \mathrm{~b}-\mathrm{h}$, respectively. The raw spectra appeared homogeneous, as can be seen by visual inspection of the data in Figure 2a. As shown in Figure 2a, the spectral curves are relatively flat between 450 and $500 \mathrm{~nm}$, but the raw spectra between 500 and $600 \mathrm{~nm}$ show significantly different features and a notable decrease in the green range around $550 \mathrm{~nm}$ with increased anthocyanin content.

\section{Statistical values of properties of interest}

The statistics of the measured anthocyanin content for the 114 P. cerasifera leaf samples determined in this study are listed in Table 1 and include the minimum, maximum, mean, standard deviation (S.D.), and number of samples for the different data sets. The reference values of anthocyanin content exhibited a broad range of variation, a result that facilitated calibration.

\section{PCR models}

PCR analysis was applied for the calibration and prediction of anthocyanin content. Eight different models for anthocyanin content were developedwith different spectra. Different PCs were applied to build the optimal calibration models. The prediction results of the calibration and validation sets are shown in Table 2. Comparison of these models show that the spectra preprocessed by SNV displayed the best performance for anthocyanin content prediction. The values of $\mathrm{R}_{\text {val }}^{2}, \mathrm{RMSE}_{\mathrm{p}}$, and $\mathrm{RPD}_{\mathrm{val}}$ in the validation set from the optimal PCR model were 0.888 , 0.315 , and 2.988 , respectively. This prediction accuracy was therefore classified as very good. The 
290

291

292

293

294

295

296

297

298

299

300

301

302

303

304

305

306

307

308

309

310

performances using SG and Raw were poor, with the $\mathrm{R}_{\text {val }}^{2}$ and $\mathrm{RPD}_{\mathrm{val}}$ for both models that were

lower than 0.80 and 2.0, respectively. According to the aforementioned criteria, we can only say that these two models might be of some value in quantitative prediction of anthocyanin content.

However, the other five PCR models yielded $\mathrm{RPD}_{\text {val }}$ values above 2.5 and the $\mathrm{R}^{2}$ val values in the range of $0.80 \leqslant \mathrm{R}^{2}<0.90$, which indicated the suitability of these models for very good quantitative predictions of leaf anthocyanin content. Figure $3 \mathrm{a}$ shows the reference versus predicted value plots for anthocyanin content using the optimal PCR model. The closer the distance the sample points are to this solid line represents better predictive results. As indicated in Figure 3a, the sample points in the calibration and validation sets were distributed near, but not tightly close to the ideal line. Also, several dots were lovated far from the ideal line, indicating a large predictive error.

\section{PLSR models}

Partial least squares regression (PLSR) models using the pretreatment spectra were also tested and the results are shown in Table 3. According to the results, the optimal preprocessing for anthocyanin content also was SNV, based on the values of the prediction performance evaluation indices. The values of the optimal determination coefficients $\mathrm{R}_{\mathrm{val}}^{2}, \mathrm{RMSE}_{\mathrm{p}}$, and $\mathrm{RPD}_{\mathrm{val}}$ for the validation set were respectively $0.901,0.259$ and 3.191 . This prediction accuracy was classified as excellent. The performance using MSC+1-Der was the worst of the tested models, with the smallest predicted $\mathrm{R}^{2}$ val and $\mathrm{RPD}_{\mathrm{val}}$ values and the largest $\mathrm{RMSE} \mathrm{p}_{\mathrm{p}}$ values. Overall, the $\mathrm{RPD}_{\mathrm{val}}$ values above 2.0 and the $\mathrm{R}_{\text {val }}^{2}$ values above 0.8 for all PLSR models indicated that these models provide very good quantitative predictions for leaf anthocyanin content. The plot of reference 
311

312 313

314 315

versus predicted values for anthocyanin content using the optimal PLSR model is shown in Figure

3b. The sample points in the calibration and validation sets are distributed much closer to the ideal line, but there was still a large deviation between the predicted values and the actual value in the PLSR models. Although according to the evaluation criteria, the optimal PLSR model should be an excellent model/predictor, the results showed that it was not ideal for use in practical analysis.

\section{BPNN models}

The performance of BPNN models was next validated using the validation set, and the prediction results are shown in Table 4 and Figure 4. As shown in Table 4, the values of $\mathrm{R}^{2}$ val, $R M S E_{p}$, and $R P D_{v a l}$ in the validation set were $0.922,0.270$, and 3.489, respectively, for the BPNNLVs model and 0.920, 0.274, and 3.439, respectively, for the BPNN-PCs model. Based on these values, both models showed excellent prediction accuracy. Very small differences in $\mathrm{R}^{2}, \mathrm{RMSE}_{\mathrm{p}}$ and RPD values were observed between the BPNN-LVs model and the BPNN-PCs model. The performance of the BPNN-LVs model was a little better than that of the BPNN-PCs model. The plots of reference versus predicted values points for anthocyanin content using the BPNN models are shown in Figure 4. The sample plots were tighter about the ideal line than those obtained using the PCR and PLSR models (see in Figure 3). The results show that the BPNN models outperformed the PCR and PLSR models, with very good agreement between the predicted values and the actual values in the BPNN models. This high prediction precision could satisfy the accuracy standards for practical applications and these results should support further research of in-field detection methods for anthocyanin content in plant leaves.

\section{Discussion}


the green range around $550 \mathrm{~nm}$ with increase of anthocyanin content. The main spectral feature of

Merzlyak \& Chivkunova (2001) that the peak magnitude was closely related to anthocyanin

content. In this study, three calibration methods were tested using all of the spectral reflectance of

the selected wavebands to build models. The selected wavebands should be sensitive to the

anthocyanin, and insensitive to chlorophyll, water, and the effects of leaf structure, and the

wavebands between 450 and $600 \mathrm{~nm}$ meet this requirement. The study results showed that spectral

reflectance between 450 and $600 \mathrm{~nm}$ well-predicted leaf anthocyanin content in P. cerasifera.

Other studies have also used the visible wavelength bands to predict leaf anthocyanin content (e.g.

Gitelson, Merzlyak \& Chivkunova, 2001; Gitelson et al., 2006; Steele et al., 2009; Garriga et al.,

2014).

In addition, as shown in Tables 2 and 3, comparison of the results using the same

pretreatments in the PCR and PLSR models, the difference values of $\mathrm{R}^{2}, \mathrm{RMSE}$, and RPD were

greater than $0.05,0.06$, and 0.8 , respectively, for the calibration set and predicted values of most

models. The better results for the calibration set indicate that the calibration model was not very

stable. The sample points for the calibration and validation sets of the PLSR model are distributed

much closer to the ideal line than those of the PCR model (Figures 3a and 3b), indicating that the

PLSR model outperformed the PCR model. Comparison of the prediction results of PCR and PLSR

models with the same pretreatment reveals better performance of PLSR models compared to that 
353 Sickman, 2008). This may be because the PLSR model can simultaneously consider the spectral 354 data matrix (X) and the target chemical properties matrix (Y) (Liu and Liu, 2013). Of the BPNN 355 models, the performance of the BPNN-LVs model was a little better than that of the BPNN-PCs

356

357

358

359

360

361

362

363

364

365

366

367

368

369

370

371

372

373

model. Mouazen et al. (2010) reported similar results for the prediction of selected soil properties

using Vis/NIR spectroscopy.

Both the leave-one-out cross-validation and predictive results showed that the BPNN model outperformed the PCR and PLSR models (Tables 2, 3 and 4, and Figures 3 and 4). The result is consistent with results from other studies of VNIRS of predictions for total anthocyanin content in new-season red-grape homogenates with PLSR and ANN (Janik et al., 2007). Additionally, Liu et al. (2008) reported similar results for the determination of acetolactate synthase activity and protein content of oilseed rape (Brassica napus L.) leaves using Vis/NIR spectroscopy. Janik, Forrester \& Rawson (2009) and Mouazen et al. (2010) also reported similar results for the prediction of selected soil chemical and physical properties using mid-infrared or Vis/NIR spectroscopy. The higher performance of the BPNN model may be because it can the nonlinear relationship typical of spectrum analysis, while PLSR and PCR models, which are built upon a linear algorithm, do not consider certain latent nonlinear information in the spectral data (Li and $H e, 2010)$. The performance of the BPNN-LVs model was a little better than that of the BPNNPCs model according to the $\mathrm{R}^{2}, \mathrm{RMSE}_{\mathrm{p}}$, and RPD values. Mouazen et al. (2010) reported similar results for the prediction of selected soil properties using Vis/NIR spectroscopy. Thus, we have demonstrated the feasibility of using spectral reflectance between 450 and $600 \mathrm{~nm}$ to estimate leaf anthocyanin content in P. cerasifera under laboratory conditions. Of cause, the canopy architecture 
374 of plants may be very complex under field conditions. In future work, additional samples and

375 samples of different species samples should be prepared for calibration based on both laboratory

376 and field conditions to expand testing of the BPNN-LVs model and improve model stability for

377 future practical applications. Additionally, chlorophyll's interference should be considered for

378 samples with low to moderate anthocyanin content (Gitelson, Chivkunova \& Merzlyak, 2009).

379 Future work could be done to discover useful information or effective wavelengths or wavebands

380 for the non-destructive determination of anthocyanin content of plants.

381

382

383

384

385

386

387

388

389

390

391

392

393

394

\section{Conclusions}

The anthocyanin content was successfully determined by spectral reflectance between 450 and $600 \mathrm{~nm}$ combined with chemometric methods. In the PCR and PLS models, spectra the preprocessed by SNV achieved the best performance for the prediction of anthocyanin content. Acceptable prediction accuracies were achieved by the PCR and PLS models, but this level of accuracy may be not satisfactory for practical applications. The performance of the PLSR models was better than that of the PCR models, but the BPNN models showed greatly improved predictive capacity. The two BPNN models were developed for the prediction of anthocyanin content outperformed the PCR and PLSR models. The $\mathrm{R}_{\text {val, }}^{2} \mathrm{RMSE}_{\mathrm{p}}$, and $\mathrm{RPD}_{\mathrm{val}}$ values for the validation set using the BPNN-LVs model were 0.922, 0.270, and 3.489, respectively, and those of the BPNN-PCs model were $0.920,0.274$, and 3.439 , respectively. Thus, the performance of the BPNN-LVs model was best. The results indicate that visible spectroscopy combined with BPNN calibrations can successfully determine the leaf anthocyanin content in P. cerasifera. Based on the results achieved in this study, it is recommended to adopt BPNN-LVs analysis as the best modeling

Peer) reviewing PDF | (2019:07:39108:1:0:NEW 29 Sep 2019) 
395

396

397

398

399

400

401

402

403

404

405

406

407

408

409

410

411

412

413

414

415

416

method to predict plant leaf anthocyanin content. The use of spectral reflectance data between 450

and $600 \mathrm{~nm}$ here represents a significant contribution to methods for the nondestructive determination of leaf total anthocyanin content.

\section{Acknowledgements}

We thank Xiaoxing Wang and Li Wang of Northwest A\&F University for assistance in examining and measuring specimens.

\section{References}

Arana I, Jaren C, Arazuri S. 2005. Maturity, variety and origindetermination in white grapes (Vitis vinifera L.) using nearinfrared reflectance technology. Journal of Near Infrared Spectroscopy 13(1): 349-357 DOI: 10.1255/jnirs.566.

Atkinson PM. 1997. Neural networks in remote sensing. International Journal of Remote Sensing 18(4): 699-709 DOI 10.1080/014311697218700.

Atzberger C, Guérif M, Baret F, Werner W. 2010. Comparative analysis of three chemometric techniques for the spectroradiometric assessment of canopy chlorophyll content in winter wheat. Computers and Electronics in Agriculture 73(2): 165-173 DOI 10.1016/j.compag.2010.05.006.

Bao YD, Kong WW, Liu F, Qiu ZJ, He Y. 2012. Detection of glutamic acid in oilseed rape leaves using near infrared spectroscopy and the least squares-support vector machine. International Journal of Molecular Sciences 13(11): 14106-14 DOI 10.3390/ijms131114106.

Blackburn GA. 2007. Hyperspectral remote sensing of plant pigments. Journal of Experimental Botany 58(4): 855-867 DOI 10.1093/jxb/erl123. 
417 Brown DJ, Bricklemyer RS, Miller PR. 2005. Validation requirement for diffuse reflectance soil 418 characterization models with a case study of VNIR soil C prediction in Montana. Geoderma 129(3): 251-267 DOI 10.1016/j.geoderma.2005.01.001.

420 Chalker-Scott L. 1999. Environmental significance of anthocyanins in plant stress responses. 421 Photochemistry and Photobiology 70(1): 1-9 DOI 10.1111/j.1751-1097.1999.tb01944.x.

422 Chu XL, Yuan HF, Lu WZ. 2004. Progress and application of spectral data pretreatment and 423 wavelength selection methods in NIR analytical technique. Progress in Chemisty 16: 528-542 DOI 424 10.3321/j.issn:1005-281X.2004.04.008.

425 Close DC, Beadle CL. 2003. The ecophysiology of foliar anthocyanin. The Botanical review 426 69(2): 149-161 DOI 10.1663/0006-8101(2003)069[0149:TEOFA]2.0.CO;2.

Despagne F, Massart DL. 1998. Neural networks in multivariate calibration. Analyst 123(11): 157R-178R. DOI 10.1039/A805562I.

Demuth H, Beale M, Hagan M. 2010. Neural Network Toolbox TM 6 User's Guide. Farifteh, J., 430 Meer, F.V., Atzberger, C., Carranza, E.J.M., 2007. Quantitative analysis of salt-affected soil reflectance spectra: a comparison of two adaptive methods (PLSR and.ANN). Remote Sensing of Environment 110: 59-78.

Du CW, Ma ZY, Zhou JM, Keith WG. 2013. Application of mid-infrared photoacoustic spectroscopy in monitoring carbonate content in soils. Sensors and Actuators B: Chemical 188: 1167-1175 DOI 10.1016/j.snb.2013.08.023.

Fennema OR. 1996. Food Chemistry. New York: Marcel Dekker, Inc.

437 Gamon JA, Surfus JS. 1999. Assessing leaf pigment content and activity with a reflectometer. 438 New Phytologist 143(1): 105 - 117 DOI 10.1046/j.1469-8137.1999.00424.x 
440 for biophysical variable estimation from remotely sensed images. IEEE Geoscience and Remote 441 Sensing Letters 3(3): 339-343. DOI.org/10.1109/LGRS.2006.871748.

442 Garriga M, Retamales J, Romero S, Caligari P, Lobos GA 2014. Chlorophyll, anthocyanin, 443 and gas exchange changes assessed by spectroradiometry in Fragaria Chiloensis under salt stress. 444 Journal of Integrative Plant Biology 56: 505-515 DOI 10.1111/jipb.12193.

445 Gitelson AA, Merzlyak MN. 2004. Non-destructive assessment of chlorophyll carotenoid and 446 anthocyanin content in higher plant leaves: principles and algorithms. p. 78-94. In: Stamatiadis S, 447 Lynch JM, Schepers JS (ed), Remote Sensing for Agriculture and the Environment. Greece: Ella. 448 Gitelson A, Keydan GP, Merzlyak MN, Gitelson C. 2006. Three-band model for noninvasive estimation of chlorophyll, carotenoids, and anthocyanin cntents in higher plant leaves. 450 Geophysical Research Letters 33(11): 431-433 DOI 10.1029/2006GL026457.

451 Gitelson AA, Merzlyak MN, Chivkunova OB. 2001. Optical properties and nondestructive 452 estimation of anthocyanin content in plant leaves. Photochemistry and photobiology 74(1): 38-45 DOI 10.1562/0031-8655(2001)0740038opaneo2.0.co2.

Gitelson AA, Chivkunova OB, Merzlyak MN. 2009. Nondestructive estimation of anthocyanins

455 and chlorophylls in anthocyanic leaves. American journal of botany 96: 1861-1868 DOI 10.3732/ajb.0800395.

Goetz AFH. 2009. Three decades of hyperspectral remote sensing of the Earth: A personal view. Remote Sensing of Environment 113(SUPPL.1): S5-S16. https://doi.org/10.1016/j.rse.2007.12.014.

Gomes V, Fernandes A, Martins-Lopes P, Pereira L, Faia AM, Melo-Pinto P. 2017. Characterization of neural network generalization in the determination of $\mathrm{pH}$ and anthocyanin content of wine grape in new vintages and varieties. Food Chemistry 218: 40-46 DOI 
463 10.1016/j.foodchem.2016.09.024.

464 Gould K, Davies K, Winefield C. 2009. Anthocyanins: biosynthesis, functions, and applications.

465 New York(NY): Springer.

466 Gould K, Kuhn DN, Lee DW, Oberbauer S. 1995. Why leaves are sometimes red. Nature. 467 378(6554): 241-242 DOI 10.1038/378241b0.

468 Gould K, McKelvie K, Markham KR. 2002. Do anthocyanins function as antioxidants in leaves? 469 imaging of $\mathrm{H} 2 \mathrm{O} 2$ in red and green leaves after mechanical injury. Plant Cell and Environment 470 25(10) : 1261-1269 DOI 10.1046/j.1365-3040.2002.00905.x.

He Y, Feng SJ. Deng XF, Li XL. 2006. Study on lossless discrimination of varieties of yogurt using the Visible/NIR-spectroscopy. Food Research International 39(6): 645-650 DOI 10.1016/j.foodres.2005.12.008.

Hu XY. 2013. Application of visible/near-infrared spectra in modeling of soil total phosphorus. Pedosphere 23(4): 417-421 DOI 10.1016/S1002-0160(13)60034-X.

Iwashina T. 2000. The structure and distribution of the flavonoids in plants. Journal of Plant Research 113(3): 287-299 DOI 10.1007/PL00013940.

478 Janik LJ, Cozzolino D, Dambergs R, Cynkar W, Gishen M. 2007. The prediction of total anthocyanin concentration in red-grape homogenates using visible-near-infrared spectroscopy and 480 artificial neural networks. Analytica chimica acta 594(1): 107-118 DOI 10.1016/j.aca.2007.05.019.

Janik L, Forrester ST, Rawson AJ. 2009. The prediction of soil chemical and physical properties from mid-infrared spectroscopy and combined partial least-squares regression and neural networks (PLS-NN) analysis. Chemometrics and Intelligent Laboratory Systems 97(2): 179-188 DOI 485 10.1016/j.chemolab.2009.04.005. 
486 Karageorgou P, Manetas Y. 2006. The importance of being red when young: anthocyanins and 487 the protection of young leaves of Quercus coccifera from insect herbivory and excess light. Tree 488 Physiology 26(5): 613-21 DOI 10.1093/treephys/26.5.613.

489 Keiner LE, Yan XH. 1998. A neural network model for estimating sea surface chlorophyll and 490 sediments from thematic mapper imagery. Remote Sensing of Environment 66(2): 153-165 DOI: 491 10.1016/S0034-4257(98)00054-6.

492 Kinoshita R, Moebius-Clune BN, van Es HM, Hively WD, Bilgili AV. 2011. Strategies for soil 493 quality assessment using visible and near-infrared reflectance spectroscopy in a western kenya 494 chronosequence. Soil Science Society of America Journal 76(6): 1776-1788 DOI 495 10.2136/sssaj2011.0307.

496

497

Kira O, Linker R, Gitelson A. 2015. Non-destructive estimation of foliar chlorophyll and carotenoid contents: Focus on informative spectral bands. International Journal of Applied Earth Observation and Geoinformation 38: 251-260 DOI: $\quad$ 10.1016/j.jag.2015.01.003.

\section{Lai B, Du LN, Hu B, Wang D, Huang XM, Zhao JT, Wang HC, Hu GB. 2019. Characterization} of a novel litchi R2R3-MYB transcription factor that involves in anthocyanin biosynthesis and tissue acidification. BMC Plant Biology 19(62): 1-13 DOI 10.1186/s12870-019-1658-5.

\section{Liakopoulos G, Nikolopoulos D, Klouvatou A, Vekkos KA, Manetas Y, Karabourniotis G.} 2006. The photoprotective role of epidermal anthocyanins and surface pubescence in young leaves of grapevine (Vitis vinifera). Annals of Botany 98(1): 257-65 DOI 10.1093/aob/mcl097.

Li XL, He Y. 2010. Evaluation of least squares support vector machine regression and other multivariate calibrations in determination of internal attributes of tea beverages. Food and Bioprocess Technology 3(5): 651-661 DOI 10.1007/s1 1947-008-0101-y.

Liu F, Zhang F, Jin ZL, He Y, Fang H, Ye QF, Zhou WJ. 2008. Determination of acetolactate 
509 synthase activity and protein content of oilseed rape (Brassica napus L.) leaves using visible/near-

510 infrared spectroscopy. Analytica chimica acta 629(1-2): 56-65 DOI 10.1016/j.aca.2008.09.027.

511 Liu XM, Liu JS. 2013. Measurement of soil properties using visible and short wave-near infrared

512 spectroscopy and multivariate calibration. Measurement 46(10): 3808-3814 DOI 513 10.1016/j.measurement.2013.07.007.

514 Liu XY, Shen J, Chang QR, Yan L, Gao YQ, Xie F. 2015. Prediction of anthocyanin content in 515 peony leaves based on visible/near-infrared spectra. Transactions of the Chinese Society for 516 Agricultural Machinery 46: 319-324 DOI 10.6041/j.issn.1000-1298.2015.09.047.

517 Lobos GA, Matus I, Rodríguez A, Romero S, Araus JL, Pozo AD. 2014. Wheat genotypic 518 variability in grain yield and carbon isotope discrimination under mediterranean conditions 519 assessed by spectral reflectance. Journal of Integrative Plant Biology 56: 470-479 DOI $520 \quad 10.1111 /$ jipb.12114.

521 Manjunath KR, Shibendu SR, Dhaval V. 2016. Identification of indices for accurate estimation 522 of anthocyanin and carotenoids in different species of flowers using hyperspectral data. Remote 523 Sensing Letters 7(10): 1004-1013 DOI 10.1111/jipb.12114.

524 Martens H, Naes T. 1989. Multivariate calibration. Wiley, New York, USA.

525 Merzlyak MN, Solovchenko A, Gitelson A. 2003. Reflectance spectral features and non526 destructive estimation of chlorophyll, carotenoid and anthocyanin Content in apple fruit. 527 Postharvest Biology and Technology 27(2): 197-211DOI 10.1016/S0925-5214(02)00066-2.

528 Merzlyak MN, Chivkunova OB, Solovchenko A, Naqvi KR. 2008. Light absorption by 529 anthocyanins in juvenile, stressed and senescing leaves. Journal of Experimental Botany 59(14): 530 3903-11DOI 10.1093/jxb/ern230.

531 Mirzaie M, Darvishzadeh R, Shakiba A, Matkan AA, Atzberger C, Skidmore A. 2014. 
532 Comparative analysis of different uni- and multi-variate methods for estimation of vegetation

533 water content using hyper-spectral measurements. International Journal of Applied Earth

534 Observation and Geoinformation 26(1): 1-11 DOI 10.1016/j.jag.2013.04.004.

535 Mouazen AM, Kuang B, Baerdemaeker JD, Ramon H. 2010. Comparison among principal

536 component, partial least squares and back propagation neural network analyses for accuracy of

537 measurement of selected soil properties with visible and near infrared spectroscopy. Geoderma

538 158(1-2): 23-31 DOI 10.1016/j.geoderma.2010.03.001.

539 Mulla DJ. 2013. Twenty five years of remote sensing in precision agriculture: Key advances and 540 remaining knowledge gaps. Biosystems Engineering 114(4): 358-371.

541 https://doi.org/10.1016/j.biosystemseng.2012.08.009.

542 Nagy A, Riczu P, Tamás J. 2016. Spectral evaluation of apple fruit ripening and pigment content 543 alteration. Scientia Horticulturae 201: 256-264.

544 Neill SO, Gould K. 1999. Optical properties of leaves in relation to anthocyanin concentration 545 and distribution. Canadian Journal of Botany 77(12): 1777-1782 DOI 10.1139/b99-153.

546 Saeys W, Mouazen AM, Ramon H. 2005. Potential for onsite and online analysis of pig manure 547 using visible and near infrared reflectance spectroscopy. Biosystems Engineering 91(4): 393-402 548 DOI 10.1016/j.biosystemseng.2005.05.001.

549 Saure MC. 1990. External control of anthocyanin formation in apple. Scientia Horticulturae 550 42(3): 181-218 DOI 10.1016/0304-4238(90)90082-P.

551 Sims DA, Gamon J. 2002. Relationships between leaf pigment content and spectral reflectance 552 across a wide range of species, leaf structures and development stages. Remote Sensing of 553 Environment 81(2-3): 337-354 DOI 10.1016/S0034-4257(02)00010-X.

554 Solovchenko AE, Merzlyak MN. 2008. Screening of visible and UV radiation as a 
555 photoprotective mechanism in plants. Russian Journal of Plant Physiology 55(6): 719-737 DOI

$55610.1134 / \mathrm{S} 1021443708060010$.

557 Solovchenko A, Chivkunova OB, Merzlyak MN, Reshetnikova IV. 2001. A

558 spectrophotometric analysis of pigments in apples. Russian Journal of Plant Physiology 48(5):693-

559700 DOI 10.1023/A:1016780624280.

560 Soriano A, Pérez-Juan PM, Vicario A, Gonzalez JM, Pérez-Coello MS. 2007. Determination

561 of anthocyanins in red wine using a newly developed method based on Fourier transform infrared

562 spectroscopy. Food Chemistry 104(3): 1295-1303 DOI 10.1016/j.foodchem.2006.10.011.

563 Steele MR, Gitelson A, Rundquist DC, Merzlyak MN. 2009. Nondestructive estimation of

564 anthocyanin content in grapevine leaves. American Journal of Enology and Viticulture 60(1): 87-

56592 DOI 10.1109/ICEMI.2009.5274122.

566 Strack D. 1997. Plant biochemistry. p. 387-416. In: harborne, J.B., P.M. Dey (ed), Phenolic

567 Metabolish. London: Academic Press.

568 Ustin SL, Gitelson AA, Jacquemoud S, Schaepman M, Asner GP, Gamon JA, Zarco- Ustin

569 SL, Gitelson A, Jacquemoud S, Pablo J, Schaepman ME, Asner GP, Gamon J, Zarco-Tejada

570 PJ. 2009. Retrieval of foliar information about plant pigment systems from high resolution

571 spectroscopy. Remote Sensing of Environment 113: S67-S77 DOI 10.5167/uzh-23317.

572 Van Den Berg A, Perkins TD. 2005. Nondestructive estimation of anthocyanin content in autumn

573 sugar maple leaves. HortScience. 40(3): 685-686.

574 Vasques GM, Grunwald S, Sickman JO. 2008. Comparison of multivariate methods for 575 inferential modeling of soil carbon using visible/near-infrared spectra. Geoderma 146(1-2):14-25

576 DOI 10.1016/j.geoderma.2008.04.007.

577 Viña A, Gitelson AA. 2005. New developments in the remote estimation of the fraction of 
578 absorbed photosynthetically active radiation in crops. Geophysical Research Letters 32(17): 195579221 DOI 10.1029/2005GL023647.

580 Viscarra Rossel RA, McGlynn RN, McBratney AB. 2006. Determining the composition of 581 mineral-organic mixes using UV-vis-NIR diffuse reflectance spectroscopy. Geoderma 137(1582 2):70-82 DOI 10.1016/j.geoderma.2006.07.004.

584 near infrared, mid infrared or combined diffuse reflectance spectroscopy for simultaneous 585 assessment of various soil properties. Geoderma $131 \quad$ (1-2): 59-75 586 DOI.org/10.1016/j.geoderma.2005.03.007.

587

588

589

590

591

592

593

594

595

596

597

598

599

600

Wang L, Chang Q, Yang J, Zhang X, Li F 2018. Estimation of paddy rice leaf area index using machine learning methods based on hyperspectral data from multi-year experiments. PLoSONE 13(12): e0207624 DOI org/10.1371/journal.pone.0207624.

Wu D, Nie PC, Cuello J, He Y, Wang ZP, Wu HX. 2011. Application of visible and near infrared spectroscopy for rapid and non-invasive quantification of common adulterants in Spirulina powder. Journal of Food Engineering 102 (3): 278-286 DOI 10.1016/j.jfoodeng.2010.09.002.

Xiong QE, Ye Z, Yang SM, Wang XY, Li FA, Li XL, Liu F, Ni S. 2003. Plant physiology experiment course. Sichuan Science \& Technology Publishing House, Chengdu.

Yang HJ, Kim HW, Kwon YS, Kim HK, Sung SH. 2017. Fast and simple discriminative analysis of anthocyanins-containing berries using LC/MS spectral data. Phytochemical Analysis 28(5): 416-423 DOI 10.1002/pca.2689.

Zeliou K, Manetas Y, Petropoulou Y. 2009. Transient winter leaf reddening in Cistus Creticus characterizes weak (stress sensitive) individuals, yet anthocyanins cannot alleviate the adverse effects on photosynthesis. Journal of Experimental Botany 60(11): 3031-3042 DOI 
$601 \quad 10.1093 / \mathrm{jxb} / \mathrm{erp} 131$.

602 Zhang YZ, Xu SZ, Cheng YW, Peng ZF, Han JM. 2018. Transcriptome profiling of 603 anthocyanin-related genes reveals effects of light intensity on anthocyanin biosynthesis in red leaf 604 lettuce. PeerJ 6:e4607 DOI 10.7717/peerj.4607.

605 Zhao C, Qu HB, Cheng YY. 2004. A new approach to the fast measurement of content of 606 amino acids in cordyceps sinensis by ANN-NIR. Spectroscopy and Spectral Analysis. 24: 50-54

607 DOI 10.1016/j.jco.2003.08.015.

608

609 
Figure 1

Correlation coefficient between anthocyanin content and Spectra of $P$. cerasifera leaves.

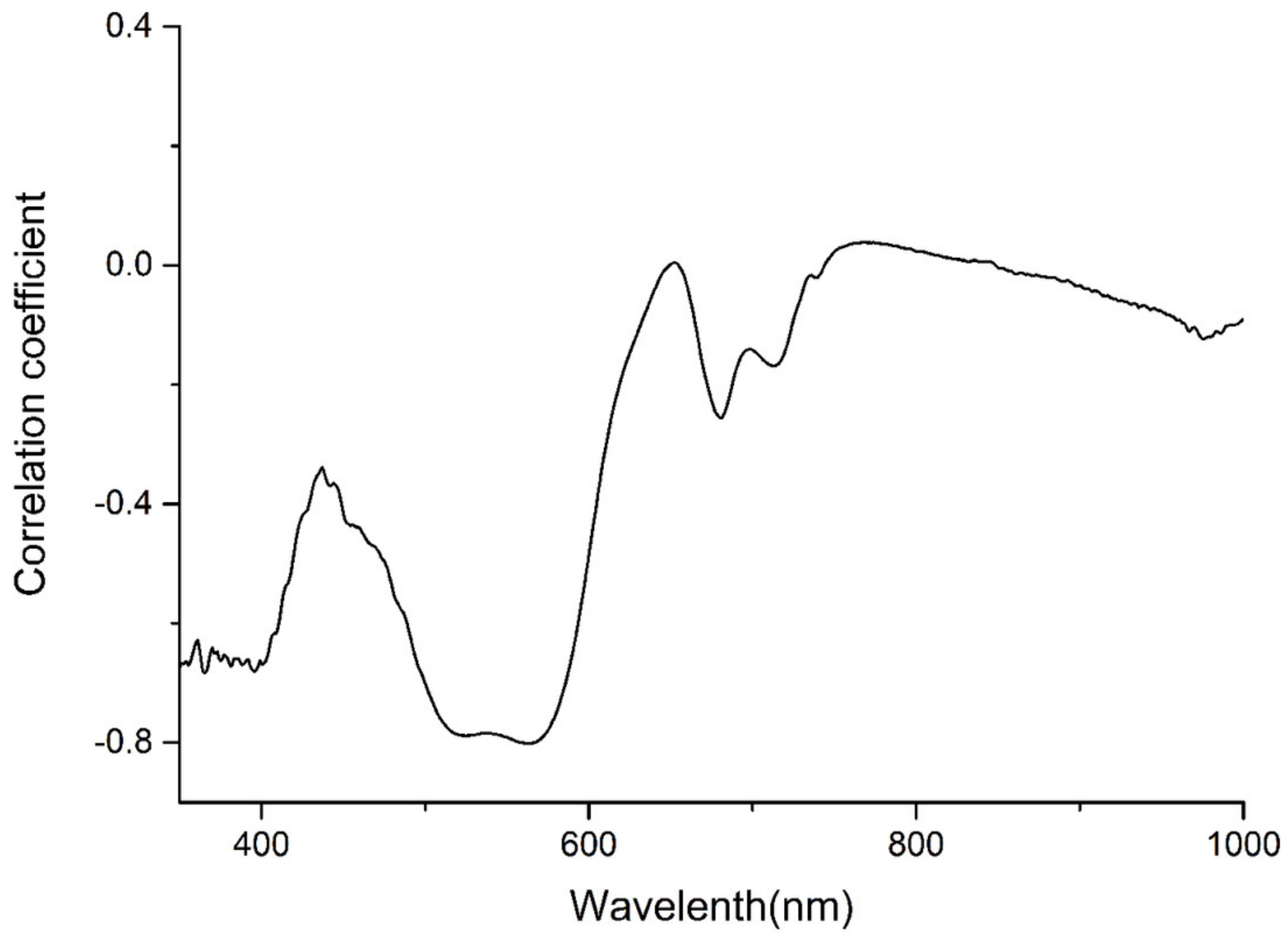


Figure 2

\section{Spectra of $P$. cerasifera leaves}

A: the raw spectra of $P$. cerasifera leaves; B: SNV; C: MSC; D: SG; E: 1-Der; F: MSC+1-Der; G: SNV+TB; H: SG+1-Der
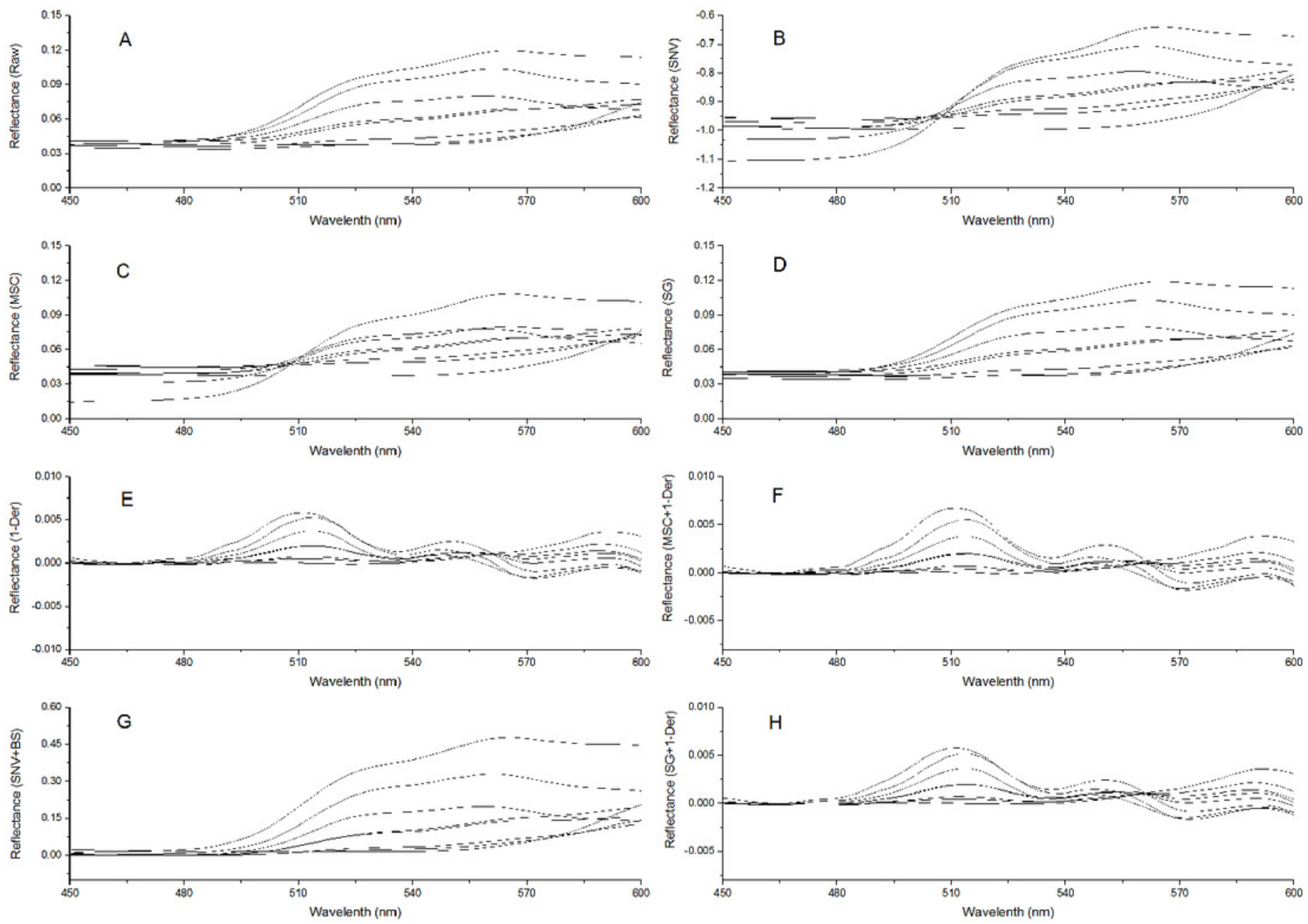
Figure 3

Measured vs. predicted values for anthocyanin content obtained by the best PCR model

(A) and PLSR model (B). Black open circles represent calibration samples and solid circles

represent validation samples. The solid lines correspond to the ideal results which meant the predicted values were equal to the reference values.
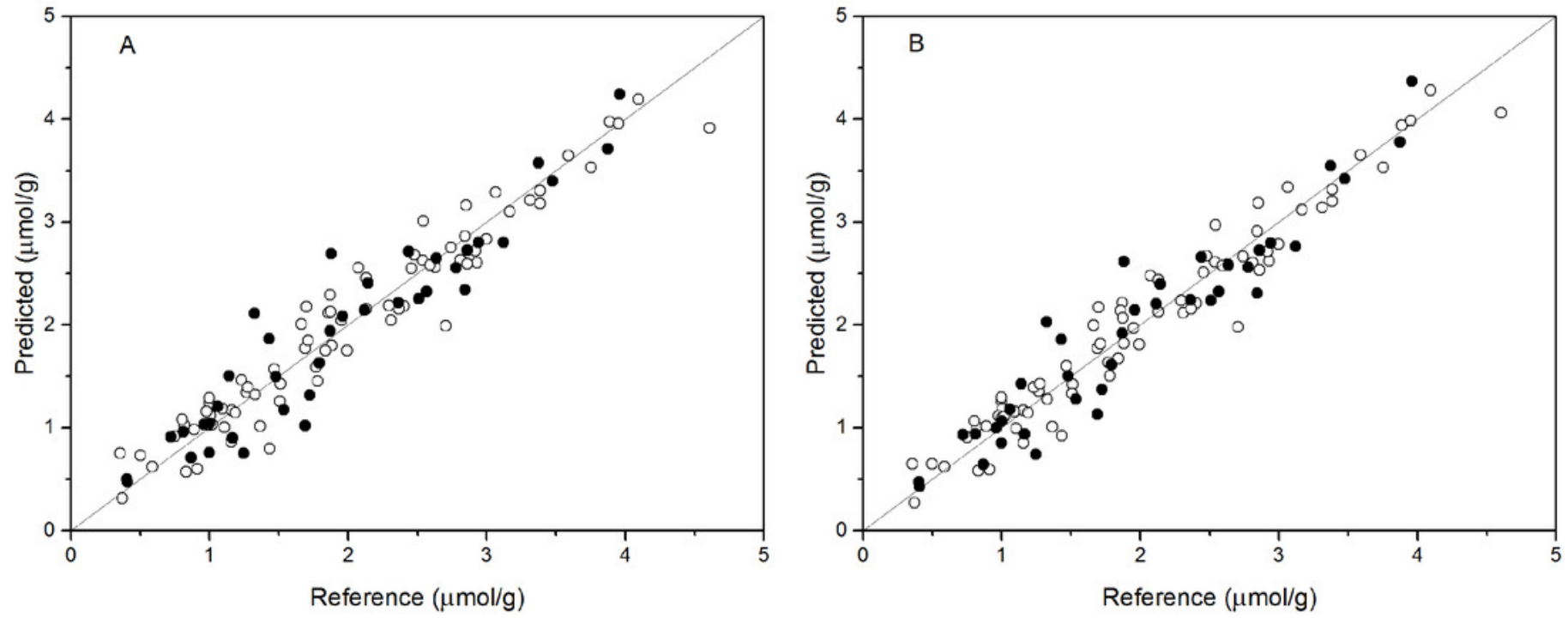
Figure 4

Measured vs. predicted values for anthocyanin content obtained by BPNN-PCs model

(A) and BPNN-LVs model (B). Black open circles represent calibration samples and solid circles represent validation samples. The solid lines correspond to the ideal results which meant the predicted values were equal to the reference values.
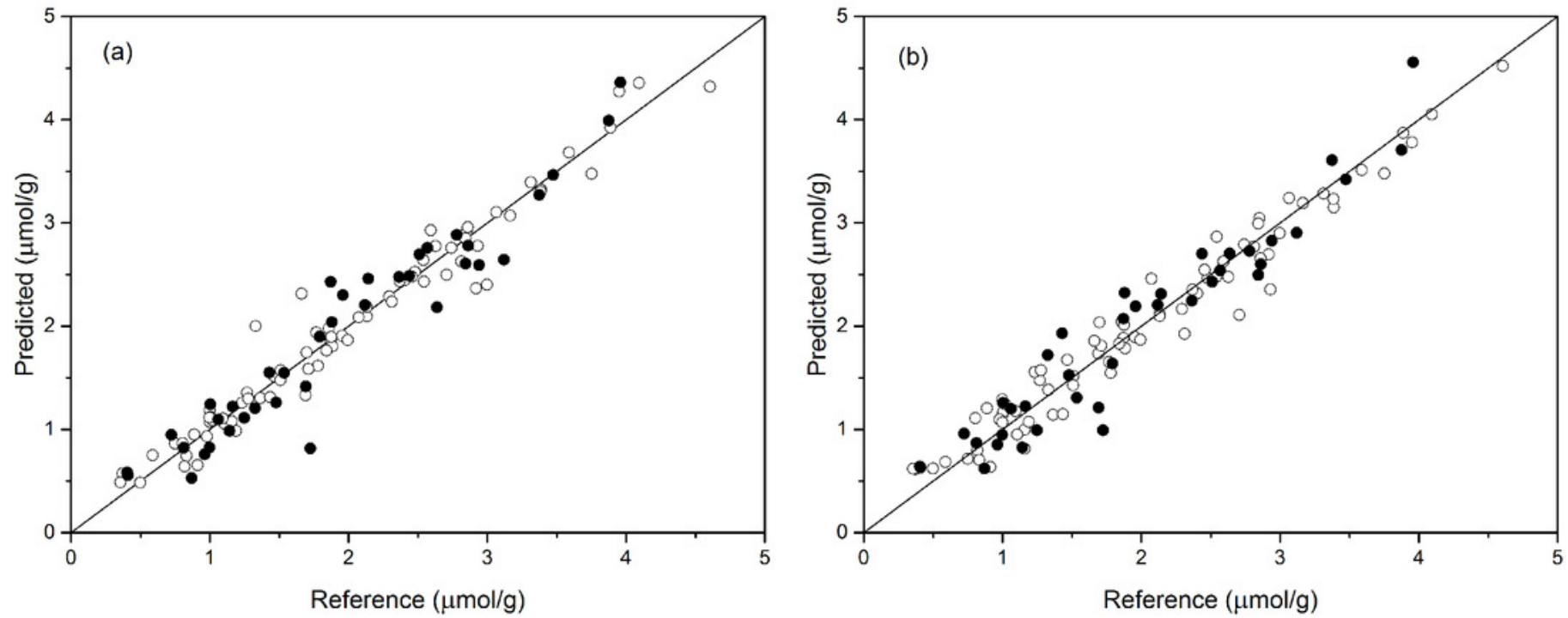


\section{Table 1 (on next page)}

The statistical values of anthocyanin content. 


\begin{tabular}{cccccc}
\hline Data sets & $\begin{array}{c}\text { Sample } \\
\text { number }\end{array}$ & Minimum & Maximum & Mean & Standard deviation \\
\hline Calibration & 76 & 0.36 & 4.61 & 1.99 & 0.98 \\
Valibration & 38 & 0.41 & 3.96 & 1.93 & 0.95 \\
All samples & 114 & 0.37 & 4.61 & 1.97 & 0.97 \\
\hline
\end{tabular}

1 
Table 2 (on next page)

Prediction results of anthocyanin content by PCR with different preprocessing in calibration and validation sets. 


\begin{tabular}{|c|c|c|c|c|c|c|c|}
\hline \multirow{2}{*}{ Pretreatment } & \multirow{2}{*}{ PCs } & \multicolumn{3}{|c|}{ Calibration } & \multicolumn{3}{|c|}{ Validation } \\
\hline & & $\mathbf{R}_{\text {cal }}^{2}$ & $\mathbf{R M S E}_{\mathbf{c}}$ & $\mathbf{R P D}_{\text {cal }}$ & $\mathbf{R}_{\text {val }}^{2}$ & $\mathbf{R M S E}_{\mathbf{p}}$ & RPD $_{\text {val }}$ \\
\hline Raw & 5 & 0.777 & 0.462 & 2.117 & 0.743 & 0.477 & 1.973 \\
\hline SNV & 5 & 0.934 & 0.250 & 3.911 & 0.888 & 0.315 & 2.988 \\
\hline MSC & 7 & 0.915 & 0.286 & 3.419 & 0.844 & 0.372 & 2.530 \\
\hline SG & 5 & 0.776 & 0.463 & 2.112 & 0.741 & 0.479 & 1.965 \\
\hline 1-Der & 6 & 0.810 & 0.427 & 2.290 & 0.843 & 0.373 & 2.523 \\
\hline MSC+1-Der & 8 & 0.881 & 0.337 & 2.902 & 0.881 & 0.337 & 2.793 \\
\hline $\mathrm{SNV}+\mathrm{BS}$ & 5 & 0.933 & 0.253 & 3.865 & 0.864 & 0.347 & 2.712 \\
\hline SG+1-Der & 8 & 0.857 & 0.370 & 2.643 & 0.864 & 0.348 & 2.705 \\
\hline
\end{tabular}

1 


\section{Table 3(on next page)}

Prediction results of anthocyanin content by PLSR with different preprocessing in calibration and validation sets. 


\begin{tabular}{|c|c|c|c|c|c|c|c|}
\hline \multirow{2}{*}{ Pretreatment } & \multirow{2}{*}{ LVs } & \multicolumn{3}{|c|}{ Calibration } & \multicolumn{3}{|c|}{ Validation } \\
\hline & & $\mathbf{R}_{\text {cal }}^{2}$ & $\mathbf{R M S E}_{\mathbf{c}}$ & $\mathbf{R P D}_{\text {cal }}$ & $\mathbf{R}_{\text {val }}^{2}$ & $\mathbf{R M S E}_{\mathbf{p}}$ & RPD $_{\text {val }}$ \\
\hline Raw & 9 & 0.933 & 0.254 & 3.850 & 0.873 & 0.336 & 2.801 \\
\hline SNV & 5 & 0.943 & 0.233 & 4.197 & 0.901 & 0.295 & 3.191 \\
\hline MSC & 4 & 0.894 & 0.318 & 3.075 & 0.847 & 0.368 & 2.558 \\
\hline SG & 9 & 0.928 & 0.262 & 3.732 & 0.878 & 0.329 & 2.861 \\
\hline 1-Der & 5 & 0.886 & 0.330 & 2.963 & 0.882 & 0.323 & 2.914 \\
\hline MSC+1-Der & 5 & 0.921 & 0.274 & 3.569 & 0.802 & 0.419 & 2.246 \\
\hline $\mathrm{SNV}+\mathrm{BS}$ & 5 & 0.943 & 0.234 & 4.179 & 0.891 & 0.311 & 3.026 \\
\hline SG+ 1-Der & 5 & 0.884 & 0.332 & 2.945 & 0.883 & 0.323 & 2.914 \\
\hline
\end{tabular}

1 


\section{Table 4 (on next page)}

Prediction results of anthocyanin content by BPNN models in calibration and validation sets. 


\begin{tabular}{lllllll}
\hline \multirow{2}{*}{ Model } & \multicolumn{2}{l}{ Calibration } & \multicolumn{3}{l}{ Validation } \\
\cline { 2 - 7 } & $\mathbf{R}_{\text {cal }}$ & $\mathbf{R M S E}_{\mathbf{c}}$ & $\mathbf{R P D}_{\text {cal }}$ & $\mathbf{R}_{\text {val }}^{\mathbf{2}}$ & $\mathbf{R M S E}_{\mathbf{p}}$ & $\mathbf{R P D}_{\text {val }}$ \\
\hline BPNN-PCs & 0.958 & 0.203 & 4.648 & 0.920 & 0.274 & 3.439 \\
BPNN-LVs & 0.961 & 0.195 & 4.819 & 0.922 & 0.270 & 3.489 \\
\hline
\end{tabular}

1 